УДК 536.629 .7

\title{
СПОСОБЫ РЕАЛИЗАЦИИ ЕДИНИЦЫ ИЗМЕРЕНИЯ ПОВЕРХНОСТНОЙ ПЛОТНОСТИ ТЕПЛОВОГО ПОТОКА
}

\author{
Ковтун С.И., канд. техн. наук, Декуша Л.В., доктор техн. наук \\ Институт технической теплофизики НАН Украины, ул. Желябова, 2а, Киев, 03680, Украина
}

\begin{abstract}
У статті розглядаються способи реалізації визначення одиниці вимірювання густини теплового потоку, що базуються на основних законах фізики, а також можливі шляхи побудови калібрувального ланцюга для забезпечення простежуваності результатів вимірювання поверхневої густини теплового потоку відповідно до вимог нового Закону України «Про метрологію та метрологічну діяльність».
\end{abstract}

В статье рассматриваются способы реализации определения единицы измерения плотности теплового потока, базирующиеся на основных законах физики, а также возможные пути построения калибровочной цепочки для обеспечения прослеживаемости результатов измерений поверхностной плотности теплового потока в соответствии с требованиями нового Закона Украины «О метрологии и метрологической деятельности».
The paper discusses providing of definition unit density of the heat flow, using basic principles of physics, and possible ways of realization a calibration chain for ensure traceability heat flux measurement accordance with the new Law of Ukraine "On metrology and metrological activity".

Библ. 11, рис. 1.

Ключевые слова: единство измерений, сенсоры теплового потока, эталон, калибровка.

\section{Постановка проблемы}

Информация об интенсивности теплового потока важна для решения множества задач, значимых в приоритетных направлениях развития науки и техники. Наиболее удобным для оценки теплового состояния и сопоставления результатов измерения является удельное значение теплового потока, а именно-его поверхностная плотность, единицей измерения которой принят $\mathrm{BT} / \mathrm{M}^{2}$.

Обеспечение единства измерений поверхностной плотности теплового потока, как и любой другой физической величины, должно включать в первую очередь нормативную базу (стандарты, методики измерения и т.д.), второй составляющей является наличие метрологических средств высшей точности - эталонов для реализации определения единицы измерения данной физической величины с установленным значением величины и связанной с ним неопределенностью измерения, которая в дальнейшем должна быть использована как основа для сравнения. Что касается нормативно-методической базы теплометрии, то здесь разработан, в том числе и сотрудниками ИТТФ, целый ряд документов, включая национальные (ДСТУ) и межгосударственные (ГОСТ), а также гармонизированы с международными (ДСТУ ISO, ДСТУ EN) стандарты Украины, регламентирующих общие требования к методам и средствам измерения тепловых величин. При этом эталонная база для средств измерения поверхностной плотности теплового потока в Украине фактически отсутствует.

\section{Анализ публикаций}

На государственном уровне вопросами единства измерений плотности теплового потока занимаются, по нашим сведениям, Национальный институт стандартов и технологий (NIST) в США и Национальная организация TNO в Нидерландах [1-4]. Так, например, в США для метрологического обеспечения тепловых измерений разработана схема калибровок сенсоров плотности теплового потока, которая показывает прослеживаемость поверяемых сенсоров (радиометров и преобразователей типа Гардона и Шмидта-Боэлтера) к национальному эталону, в качестве которого применен высокоточный криогенный радиометр (HACR) [4].

\section{Цель статьи}

Проанализировать возможные варианты реализации единицы поверхностной плотности теплового потока для обеспечения прослеживаемости результатов измерения в соответствии с требованиями Закона Украины «Про метрологію та метрологічну діяльність».

\section{Изложение основного материала}

Реализовать единицу плотности теплового потока можно несколькими способами, используя основные положения физики, рассмотренными ниже.

Согласно закону Стефана-Больцмана, плотность потока теплового излучения, поступающая от источника теплового излучения на поверхность исследуемого сенсора, определяется выражением:

$q=\varepsilon_{n p} \cdot \varphi_{1-2} \cdot \sigma \cdot\left(T_{1}^{4}-T_{2}^{4}\right)$,

где $\varepsilon_{\text {пр }}$ - приведенная степень черноты; $\varphi_{(1-2)}-$ угловой коэффициент; $\sigma$ - постоянная Стефана-Больцмана; Т температура, индексом “1” обозначена излучающая поверхность ИТИ, а индексом "2"- поверхность сенсора теплового потока.

Современный уровень развития термометрии обеспечивает высокую точность задания и измерения значений температуры $\mathrm{T}_{1}$ и $\mathrm{T}_{2}$. Постоянная СтефанаБольцмана является фундаментальной физической константой, значение которой $\sigma=5,670400 \cdot 10^{-8} \mathrm{BT} /\left(\mathrm{M}^{2} \cdot \mathrm{K}^{4}\right)$ известно с высокой точностью [5], поэтому основная погрешность измерения плотности теплового потока 
зависит от погрешности определения терморадиационных характеристик поверхностей сенсора и источника теплового излучения. В настоящее время эти значения определяются с погрешностью более $1 \%$.

В соответствии с теорией теплопроводности плотность теплового потока через слой однородного материала пропорциональна градиенту температуры (закон Фурье):

$q=-\lambda \cdot(\partial T / \partial x)=-\lambda \cdot\left(T_{2}-T_{1}\right) / h$,

где $\lambda$ - коэффициент теплопроводности слоя, участвующего в теплообмене; $\partial T / \partial x$ - градиент температуры; $h$ - толщина слоя.

Основными составляющими погрешности данного способа являются: погрешность измерения температуры и линейных размеров теплопроводящего слоя, погрешность определения коэффициента теплопроводности, которая в настоящее время для рабочих эталонов теплопроводности составляет порядка $3 \%$ [6].

Основываясь на измерении электрических величин, эталонированная плотность теплового потока может быть реализована посредством подведения электрической мощности $W$ заданного значения к источнику теплоты, имеющего конечные геометрические размеры, и определяется формулой:

$q=W / A$,
Учитывая современный уровень развития измерений электрических и геометрических величин, данный метод задания и измерения нормированного значения плотности теплового потока представляется наиболее перспективным.

При подведении теплового потока посредством конвекции с известным значением коэффициента теплообмена $\alpha$, создаваемого на поверхности преобразователя, рассчитать нормированное значение плотности теплового потока можно с применением закона Ньютона-Рихмана:

$q=\alpha \cdot \Delta T$.

Однако обеспечить точность задания и поддержания значения коэффициента теплообмена весьма затруднительно, поэтому использование данного способа для прецизионного воспроизведения единицы измерения является нецелесообразным.

Для обеспечения единства измерений необходимо иметь документально подтвержденную прослеживаемость эталона единицы измерения поверхностной плотности теплового потока к национальным эталонам, эталонам других государств или международных эталонов соответствующих единиц измерения, которая должна быть реализована через непрерывную цепочку калибровок (рис. 1).

где $A$-площадь рабочей поверхности источника теплоты.

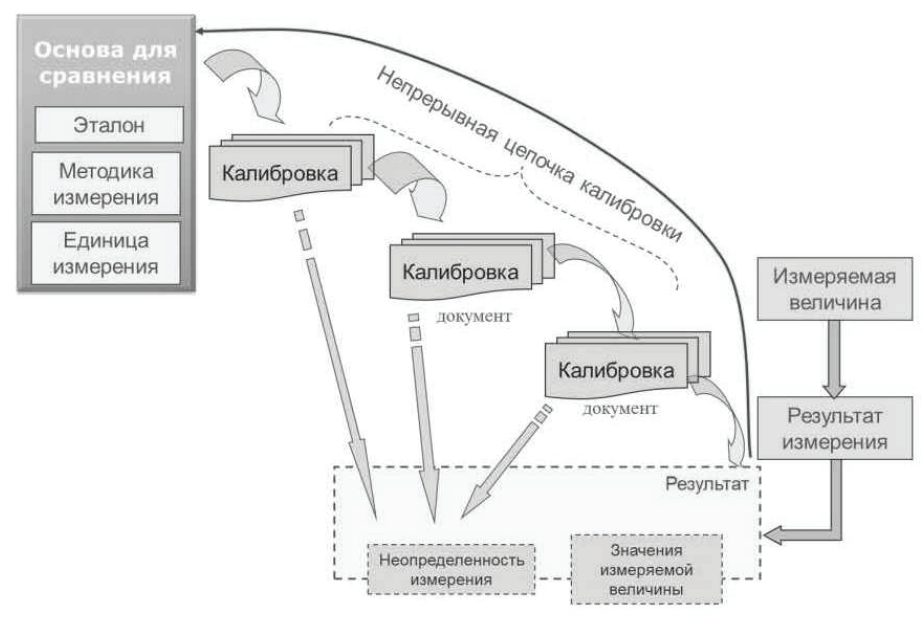

Рис. 1. Прослеживаемость результатов измерений.

В соответствии с положениями новой редакции Закона Украины «О метрологии и метрологической деятельности» калибровка - это совокупность операций, с помощью которой при заданных условиях на первом этапе, устанавливают соотношение между значениями величины с неопределенностями измерений, которые обеспечивают эталоны, и соответствующими показаниями со связанными с ними неопределенностями измерений, а на втором этапе используют эту информацию для установления соотношения для получения результата измерения из показания. В идеале неопределенность эталона не должна превышать значение, равное 1/4 неопределенности калибруемого средства измерения. В этом случае общее влияние неопределенности эталонов считается незначительным.

Наиболее близким государственным эталоном является первичный эталон единицы энергетической освещенности некогерентным излучением ДЕТУ 11-01-96, разработанный и созданный в 1996 году ННЦ «Институт метрологии» [7]. Эталон предназначен для воспроизведения и передачи единицы плотности потока оптического излучения в диапазоне от $10 \mathrm{BT} / \mathrm{M}^{2}$ до $10^{5} \mathrm{BT} / \mathrm{M}^{2}$ со средним квадратичным отклонением результата измерения, не превышающим $0,25 \cdot 10^{-2}$, при этом граница неисключенной относительной систематической погрешности не превышает $0,3 \cdot 10^{-2}$ [8]. Эталон, метроло- 
гические характеристики которого отвечают существующему мировому уровню в области оптико-физических измерений, возглавляет государственную поверочную схему для средств измерения энергетической освещенности тепловым и солнечным излучением в Украине [9]. Средством передачи единицы энергетической освещенности служит абсолютный приемник теплового излучения. Государственный эталон ДЕТУ 11-01-96 перекрывает достаточно широкий динамический диапазон и метрологически обеспечивает измерения потоков теплового излучения. Но вместе с тем, передача единицы энергетической освещенности, получаемой от государственного первичного эталона, рабочим средствам измерения, осуществляется при помощи Вторичного эталона энергетической освещенности солнечным излучением, обладающего существенно ограниченным диапазоном единицы измерения (от 400 Вт/м² до 1360 $\left.\mathrm{BT} / \mathrm{M}^{2}\right)$.

Для обеспечения единства измерений поверхностной плотности теплового потока плоскими преобразователями создан метрологический комплекс, предназначенный для воспроизведения, хранения и передачи размера соответствующей единицы измерения [10].

Метрологический комплекс состоит из эталонных средств, среди которых такие:

1) эталонная установка УВТ-1 с кондуктивным подводом тепловой энергии, реализующая способ воспроизведения единицы измерения по электрической мощности (см. формулу (3); основными узлами УВТ-1 являются: тепловой блок для обеспечения заданного режима измерения, электронный блок для задания и контроля тепловых параметров теплового блока и сбора первичной информации, эталонная мера сопротивления, а также стабилизированный блок питания и контрольно-измерительные приборы. Эталонная установка позволяет исследовать метрологические характеристики плоских сенсоров теплового потока;

2) комплект эталонных преобразователей теплового потока с улучшенными метрологическими характеристиками [11].

В результате исследований метрологических характеристик эталонной установки УВТ-1 и комплекта эталонных преобразователей теплового потока установлено, что метрологический комплекс обеспечивает воспроизведение значений единицы измерения поверхностной плотности теплового потока в диапазоне $(0,1 \ldots 20)$ кВт/м ${ }^{2}$ при температуре 300 K до 473 K со средним квадратическим отклонением результата измерений не превышающим 0,15 \% и в пределах неисключенной систематической погрешности $\pm 0,18 \%$; расширенная неопределенность составила 0,36 \%, что соответствует современному мировому уровню в области тепловых измерений.

На пути калибровки метрологического комплекса, реализующего единицу измерения поверхностной плотности теплового потока с применением государственного эталона энергетической освещенности, в виду различия способа воспроизведения тепловой энергии в эталонной установке и в ДЕТУ 11-01-96, возникают некоторые затруднения. Для реализации их сличения необходимо создавать компаратор, который позволит исследовать характеристики как плоских преобразователей так и приемников теплового излучения.

Следует отметить, что, исходя из способов реализации единицы измерения Вт/ $\mathrm{M}^{2}$, возможны альтернативные пути построения калибровочной цепочки, восходящей одновременно к нескольким государственным эталонам. В случае реализации воспроизведения единицы по закону Фурье (2) возможна привязка к государственным эталонам, обеспечивающим получение значений коэффициента теплопроводности, температуры и линейных размеров. В случае реализации воспроизведения по электрической мощности (3) - к эталонам электрических величин и линейных размеров. В итоге оценка результата измерений будет включать всю совокупность неопределенностей, которые обеспечивают каждый из задействованных в калибровочной цепи эталонов.

Для выполнения требований положений Закона Украины «О метрологии и метрологической деятельности» эталонная база Украины для метрологического обеспечения теплопоточных измерений сегодня как никогда остро нуждается в реконструкции и модернизации. Достигнутый на данный момент уровень метрологических характеристик эталонной установки и комплекта эталонных преобразователей теплового потока дает основания для рассмотрения и утверждения метрологического комплекса в качестве первичного эталона, что непременно станет весомым шагом в обеспечении единства измерений поверхностной плотности теплового потока в Украине.

\section{ЛИТЕРАТУРА}

1. Holmberg D. A progress report on the NIST convective heat flux calibration facility / David G. Holmberg, Carole A. Womeldorf // Proceedings of the 5th ASME/JSME Joint thermal engineering conference. - San Diego, California, 1999. - P.1-8.

2. Standard Test Method for Steady-State Heat Flux Measurements and Thermal Transmission Properties by Means of the Guarded-Hot-Plate Apparatus: ASTM C17710. - [Acting from 01-Sep-2010]. - ASTM International, 2010. - 23 p. - (International Standard).

3. Standard Test Method for Steady-State Thermal Transmission Properties by Means of the Heat Flow Meter Apparatus: ASTM C158-10. - [Acting from 01-Sep-2010]. ASTM International, 2010. - 15 p.-(International Standard).

4. NIST MEASUREMENT SERVICES: Heat-Flux Sensor Calibration / Benjamin K. Tsai, Charles E. Gibson, Annageri V. Murthy and others // NIST Special Publication 250-65. - 2004. - $44 \mathrm{p}$.

5. Физические величины: справочник / [Бабичев А.П., Бабушкина Н.А., Братковский А.М. [и др.]; под ред. И.С. Григорьева, Е.З. Мейлихова]. - М.: Энергоатомиздат, 1991. - 1232 с. - ISBN 5-283-04013-5.

6. Соколов Н.А. Метрологическое обеспечение 
энергосбережения (Измерение теплопроводности и свя-занных с ней величин): [учебное пособие] / Н.А. Соколов. - С.-Петерб.: НИУПЦ «Межрегиональный институт стекла», 2005. - 128 с.

7. Назаренко Л.А. Державний спеціальний еталон одиниці енергетичної освітленості некогерентним випромінюванням / Л.А. Назаренко, В.І. Полевой, Л.І. Бондаренко // УМЖ. - 1995. - Вип. 1. - С. 31-36.

8. Бондаренко Л.И., Прокопенко Н.В. Метрологическое обеспечение оптико-физических измерений в системе экологического мониторинга и контроля параметров микроклимата // Вестник ХНАДУ. - 2011. Вып. 52. - С. 162-166.

\section{WAYS OF REALIZATION OF MEASUREMENT UNITS OF THE HEAT FLUX SURFACE DENSITY}

\section{Kovtun S.I., Dekusha L.V.}

Institute of Engineering Thermophysics National Academy of Sciences of Ukraine, vul. Zhelyabova, 2a, Kiev, 03680, Ukraine

The objective of paper is analyze of possible ways of realization of the heat flux surface density units order to ensure the traceability of the measurement results in accordancewith the requirements of the Law of Ukraine "On metrology and metrological activity". Providing of definition unit density of the heat flow using basic principles of physics are discussed.

References 11, figures 1.

Key words: measurement traceability, heat flux sensors, reference, calibration.

1. David G. Holmberg, Carole A. Womeldorf. A progress report on the NIST convective heat flux calibration facility. Proceedings of the 5th ASME/JSME Joint thermal engineering conference. San Diego, California, 1999. P. 1-8. (Eng.)

2. Standard Test Method for Steady-State Heat Flux Measurements and Thermal Transmission Properties by Means of the Guarded-Hot-Plate Apparatus: ASTM C17710. [Acting from 01-Sep-2010]. ASTM International, 2010. 23 p. (International Standard). (Eng.)

3. Standard Test Method for Steady-State Thermal Transmission Properties by Means of the Heat Flow Meter Apparatus: ASTM C158-10. [Acting from 01-Sep-2010]. ASTM International, 2010. 15p. (International Standard).
9. ДСТУ 3193-95. Метрологія. Державна повірочна схема для засобів вимірювань енергетичної освітленості некогерентним випромінюванням. - На заміну ГОСТ 80195-89; чинний від 01.07.1996. - К. : Держстандарт України, 1996. - 18 с.

10. Ковтун, C.I. Еталонна установка для атестації перетворювачів теплового потоку виду допоміжної стінки: автореф. дис. ... канд. техн. наук : 05.11.04 / Ковтун Світлана Іванівна ; Нац. акад. наук України, Ін-т техн. теплофізики. - К., 2013. - 23 с.

11. Ковтун С.И., Декуша Л.В., Грищченко Т.Г. Основы проектирования эталонных преобразователей теплового потока // Промышленная теплотехника. - Т. 34, № 5. - 2012. - C. 80-90.

(Eng.)

4. Benjamin K. Tsai, Charles E. Gibson, Annageri V. Murthy and others. NIST MEASUREMENT SERVICES: Heat-Flux Sensor Calibration. NIST Special Publication. 250-65. 2004. 44 p. (Eng.)

5. Babichev A.P., Babushkina N.A., Bratkovskiy A.M. [and etc.] Physical quantities: a guide. M. Energoatomisdat, 1991. 1232 p. ISBN 5-283-04013-5. (Rus.)

6. Sokolov N.A. Metrological support of energy saving (thermal conductivity measurement and related values): tutorial. St. Petersburg, 2005. 128 p. (Rus.)

7. L.A. Nazarenko, V.I. Polevoy, L.I. Bondarenko. State special standard of unit of illumination incoherent energy radiation. UMJ. 1995. Vol. 1. P. 31-36. (Ukr.)

8. Bondarenko L.I., Prokopenko N.V. Metrological support of optical-physical measurements in the system of environmental monitoring and control of microclimate parameters. Bulletin KhNAHU. 2011. Vol. 52.P. 162-166. (Rus.)

9. NSTU 3193-95. Metrology. State verification scheme for means of measuring energy illumination incoherent radiation. K. State Standard of Ukraine, 1996. 18 p. (Ukr.)

10. Kovtun S.I. Standard facility for attestation of heat flux sensor of auxiliary wall type : Abstract of thesis ... candidate in technical sciences : 05.11.04. Institute of Technical Thermophysic of the NAS of Ukraine. K., 2013. 23 p. (Ukr.)

11. Kovtun S.I., Dekusha L.V., Grischenko T.G. Fundamentals of reference heat flow transducers. Industrial Heat Engineering. V. 34, № 5. 2012. P. 80-90. (Rus.)

Получено 19.01.2017 Received 19.01.2017 